\title{
The need for an early anti-neutrino run of $\mathrm{NO} \nu \mathrm{A}$
}

\author{
Suprabh Prakash, ${ }^{1}$ Ushak Rahaman and S. Uma Sankar \\ Department of Physics, Indian Institute of Technology Bombay, \\ Powai, Mumbai 400 076, India \\ E-mail: suprabh@phy.iitb.ac.in, ushak@phy.iitb.ac.in, \\ uma@phy.iitb.ac.in
}

ABSTRACT: The moderately large value of $\theta_{13}$, measured recently by reactor experiments, is very welcome news for the future neutrino experiments. In particular, the $\mathrm{NO} \nu \mathrm{A}$ experiment, with 3 years each of $\nu$ and $\bar{\nu}$ runs, will be able to determine the mass hierarchy if one of the following two favourable combinations is true: normal hierarchy with $-180^{\circ} \leq \delta_{\mathrm{CP}} \leq 0$ or inverted hierarchy with $0 \leq \delta_{\mathrm{CP}} \leq 180^{\circ}$. In this report, we study the hierarchy reach of the first 3 years of $\mathrm{NO} \nu \mathrm{A}$ data. Since $\sin ^{2} 2 \theta_{23}$ is measured to be non-maximal, $\theta_{23}$ can be either in the lower or higher octant. Pure $\nu$ data is affected by $\theta_{13}$-hierarchy and octanthierarchy degeneracies, which limit the hierarchy sensitivity of such data. A combination of $\nu$ and $\bar{\nu}$ data is not subject to these degeneracies and hence has much better hierarchy discrimination capability. We find that, with a 3 year $\nu$ run, hierarchy determination is possible for only two of the four octant-hierarchy combinations. Equal 1.5 year runs in $\nu$ and $\bar{\nu}$ modes give good hierarchy sensitivity for all the four combinations.

Keywords: Neutrino Physics, CP violation

ARXIV EPRINT: 1306.4125

\footnotetext{
${ }^{1}$ Present address: Harish-Chandra Research Institute, Chhatnag Road, Jhunsi, Allahabad 211019, India.
} 


\section{Contents}

1 Introduction 1

2 Degeneracies in $P\left(\nu_{\mu} \rightarrow \nu_{e}\right)$ and $P\left(\bar{\nu}_{\mu} \rightarrow \bar{\nu}_{e}\right) \quad 2$

2.1 Hierarchy- $\delta_{\mathrm{CP}}$ degeneracy 2

$2.2 \theta_{13}$-hierarchy degeneracy 3

2.3 Octant-hierarchy degeneracy 4

3 Results 4

3.1 Simulation details 4

3.2 Effect of precision of $\sin ^{2} 2 \theta_{13}$ on hierarchy determination 5

3.3 Resolving the octant-hierarchy degeneracy 5

$\begin{array}{ll}3.4 \text { Advantages of } 1.5 \nu+1.5 \bar{\nu} \text { run of } \mathrm{NO} \nu \mathrm{A} & 10\end{array}$

4 Conclusions $\quad 11$

\section{Introduction}

Neutrino oscillations are one of the most significant evidences for physics beyond standard model. The discovery by the reactor neutrino experiments during the last two years, that $\theta_{13}$ is non-zero, created a lot of excitement [1-3]. In fact, its measured value is moderately large and is just below the upper limit established earlier [4-6]. The Daya Bay experiment gives the most precise value: $\sin ^{2} 2 \theta_{13}=0.089 \pm 0.01$ [1]. By the end of Daya Bay's run, the uncertainty is expected to be reduced from the present $10 \%$ to $5 \%$ [7]. Another important recent discovery is the precision measurement of $\sin ^{2} 2 \theta_{23}$ by MINOS, which found it to be non-maximal [8]. This raises the problem of determining the true octant of $\theta_{23}$.

Neutrino oscillations depend on two mass-squared differences, $\Delta_{21}=m_{2}^{2}-m_{1}^{2}$ and $\Delta_{31}=m_{3}^{2}-m_{1}^{2}$, three mixing angles and a CP violating phase $\delta_{\mathrm{CP}}$. Here $m_{1}, m_{2}$ and $m_{3}$ are the masses of three mass eigenstates. The present oscillation data determine the mass-squared differences and mixing angles reasonably well [9-11]. The observed energy dependence of the solar neutrino survival probability requires $\Delta_{21}$ to be positive. But the present data allow $\Delta_{31}$ to be either positive or negative. The case of positive $\Delta_{31}$ is called normal hierarchy $(\mathrm{NH})$ and that of negative $\Delta_{31}$ is called inverted hierarchy (IH). If the lightest neutrino mass is negligibly small, we have the following patterns: $m_{3} \gg m_{2}>m_{1}$ for $\mathrm{NH}$ and $m_{2}>m_{1} \gg m_{3}$ for IH. It is possible that all the three masses are nearly degenerate. In such a situation also the data allows either hierarchy. Determination of the neutrino mass hierarchy, the octant of $\theta_{23}$ and the search for $\mathrm{CP}$ violation in neutrino sector are the important physics goals of current and future oscillation experiments. 
A number of models are proposed to explain the observed pattern of neutrino masses and mixing. Among these, the models predicting NH are qualitatively different from those predicting IH. Therefore, the determination of the neutrino mass hierarchy will enable us to distinguish between different types of models [12]. A large number of these models predict $\theta_{13}$ to be zero and $\theta_{23}$ to be maximal. A precise measurement of the deviations from these predictions will enable us to discern the pattern of symmetry breaking in the models. Ever since the possibility of generating baryon asymmetry via leptogenesis was raised [13], the search for leptonic CP violation has acquired great significance.

A simple way to achieve the above three goals is to measure the probabilities for $\nu_{\mu} \rightarrow$ $\nu_{e}$ oscillation $\left(P\left(\nu_{\mu} \rightarrow \nu_{e}\right)\right)$ and $\bar{\nu}_{\mu} \rightarrow \bar{\nu}_{e}$ oscillation $\left(P\left(\bar{\nu}_{\mu} \rightarrow \bar{\nu}_{e}\right)\right)$. The leading term in both these probabilities is proportional to $\sin ^{2} 2 \theta_{13} \sin ^{2} \theta_{23}$. Therefore, the moderately large value of $\theta_{13}$ makes it possible for the current experiments to address the problems of both hierarchy and the octant of $\theta_{23}$. Appreciable matter effects in the $\mathrm{NO} \nu \mathrm{A}$ experiment make it an excellent tool to determine the hierarchy for favourable values of parameters $[14,15]$. In addition, $\mathrm{T} 2 \mathrm{~K}$ and $\mathrm{NO} \nu \mathrm{A}$ can determine octant of $\theta_{23}$ at $2 \sigma[16,17]$ for all values of $\delta_{\mathrm{CP}}$.

\section{Degeneracies in $P\left(\nu_{\mu} \rightarrow \nu_{e}\right)$ and $P\left(\bar{\nu}_{\mu} \rightarrow \bar{\nu}_{e}\right)$}

Among the neutrino oscillation parameters, there are two small quantities: $\theta_{13}$ and $\alpha=$ $\Delta_{21} / \Delta_{31}$. By setting one or both to be zero, it was possible so far, to reduce all the measured survival probabilities to effective two flavour formulae. In the $\nu_{e}$ appearance measurements at $\mathrm{T} 2 \mathrm{~K}$ and $\mathrm{NO} \nu \mathrm{A}$, the first non-trivial three flavour oscillation effects will be observed, which are proportional to the small quantities $\theta_{13}$ and $\alpha$. In the approximation of keeping only the terms which are second order in these small quantities, the $\nu_{\mu} \rightarrow \nu_{e}$ oscillation probability is given by $[18,19]$,

$$
\begin{aligned}
P\left(\nu_{\mu} \rightarrow \nu_{e}\right)= & \sin ^{2} 2 \theta_{13} \sin ^{2} \theta_{23} \frac{\sin ^{2} \hat{\Delta}(1-\hat{A})}{(1-\hat{A})^{2}} \\
& +\alpha \cos \theta_{13} \sin 2 \theta_{12} \sin 2 \theta_{13} \sin 2 \theta_{23} \cos \left(\hat{\Delta}+\delta_{\mathrm{CP}}\right) \frac{\sin \hat{\Delta} \hat{A} \sin \hat{\Delta}(1-\hat{A})}{\hat{A}} \frac{\sin ^{2} \hat{\Delta} \hat{A}}{1-\hat{A}} \\
& +\alpha^{2} \sin ^{2} 2 \theta_{12} \cos ^{2} \theta_{13} \cos ^{2} \theta_{23} \frac{\hat{A}^{2}}{}
\end{aligned}
$$

Here $\hat{\Delta}=\Delta_{31} L / 4 E$ and $\hat{A}=A / \Delta_{31}$, where $A$ is the Wolfenstein matter term [20]. The expression for $P\left(\bar{\nu}_{\mu} \rightarrow \bar{\nu}_{e}\right)$ is obtained by changing the signs of $\hat{A}$ and $\delta_{\mathrm{CP}}$ in $P\left(\nu_{\mu} \rightarrow \nu_{e}\right)$. $\Delta_{31}$ is positive for $\mathrm{NH}$ and is negative for IH. From eq. (2.1), we see that the oscillation probability depends on unknowns, i.e. hierarchy, octant of $\theta_{23}$ and $\delta_{\mathrm{CP}}$, along with other parameters, such as $\theta_{13}$. A measurement of these probabilities, in general, gives rise to degenerate solutions.

\subsection{Hierarchy- $\delta_{\mathrm{CP}}$ degeneracy}

From the current measurements, we know that $\sin 2 \theta_{13} \approx 0.3$ whereas $|\alpha| \approx 0.03$. Hence, the first term in $P\left(\nu_{\mu} \rightarrow \nu_{e}\right)$ (and in $P\left(\bar{\nu}_{\mu} \rightarrow \bar{\nu}_{e}\right)$ ) is much larger than second term and the 


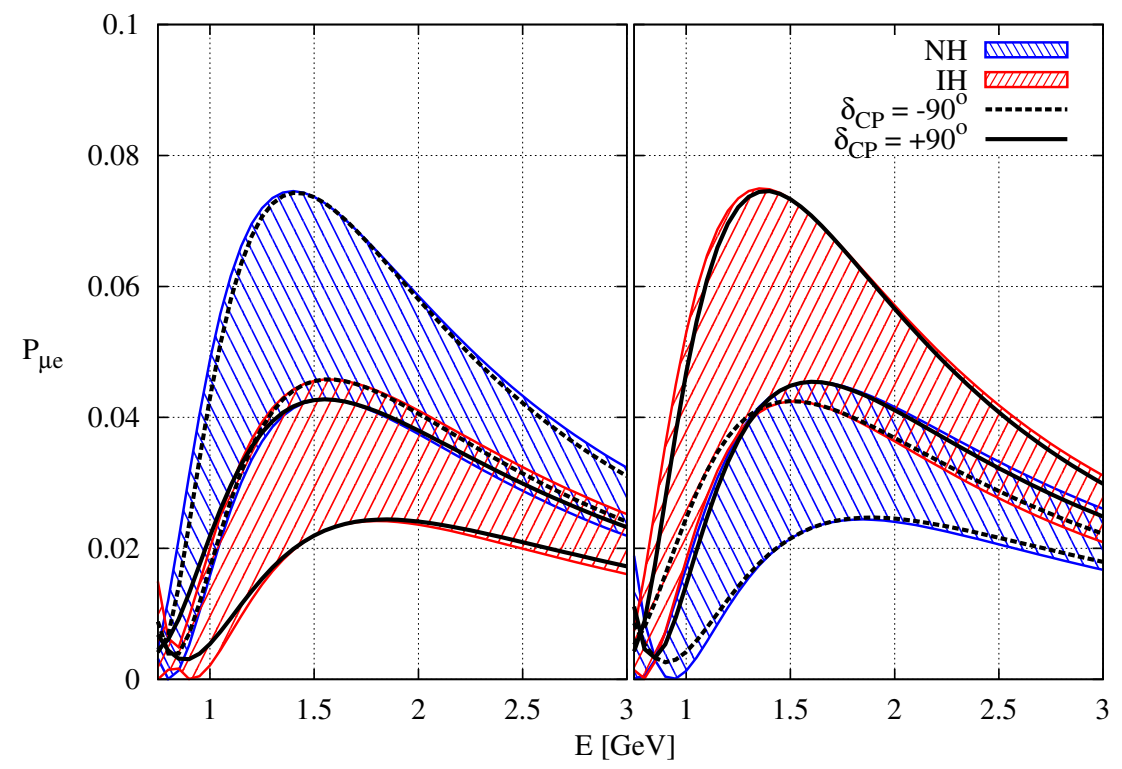

Figure 1. $P\left(\nu_{\mu} \rightarrow \nu_{e}\right)$ (left panel) and $P\left(\bar{\nu}_{\mu} \rightarrow \bar{\nu}_{e}\right)$ (right panel) vs. energy for NO $\nu$ A. Variation of $\delta_{\mathrm{CP}}$ leads to the blue (red) bands for NH (IH). The plots are drawn for maximal $\theta_{23}$ and other neutrino parameters given in the text.

third term is completely negligible. The largest amount of matter effect and hence hierarchy sensitivity, comes from the leading term. For NH (IH), the first term in $P\left(\nu_{\mu} \rightarrow \nu_{e}\right)$ becomes larger (smaller). For $P\left(\bar{\nu}_{\mu} \rightarrow \bar{\nu}_{e}\right)$, the situation is reverse. These changes in $P\left(\nu_{\mu} \rightarrow \nu_{e}\right)$ and in $P\left(\bar{\nu}_{\mu} \rightarrow \bar{\nu}_{e}\right)$ can be amplified or canceled by the second term, depending on the value of $\delta_{\mathrm{CP}}$. This is illustrated in figure 1 , where $P\left(\nu_{\mu} \rightarrow \nu_{e}\right)$ and $P\left(\bar{\nu}_{\mu} \rightarrow \bar{\nu}_{e}\right)$ are plotted for the $\mathrm{NO} \nu \mathrm{A}$ experiment. For $\mathrm{NH}$ and $\delta_{\mathrm{CP}}$ in the lower half plane (LHP) $\left(-180^{\circ} \leq \delta_{\mathrm{CP}} \leq 0\right)$, the values of $P\left(\nu_{\mu} \rightarrow \nu_{e}\right)\left(P\left(\bar{\nu}_{\mu} \rightarrow \bar{\nu}_{e}\right)\right)$ are reasonably greater (lower) than the values of $P\left(\nu_{\mu} \rightarrow \nu_{e}\right)\left(P\left(\bar{\nu}_{\mu} \rightarrow \bar{\nu}_{e}\right)\right)$ for IH and any value of $\delta_{\mathrm{CP}}$. Similarly, for IH and $\delta_{\mathrm{CP}}$ in the upper half plane (UHP) $\left(0 \leq \delta_{\mathrm{CP}} \leq 180^{\circ}\right)$ the values of $P\left(\nu_{\mu} \rightarrow \nu_{e}\right)\left(P\left(\bar{\nu}_{\mu} \rightarrow \bar{\nu}_{e}\right)\right)$ are reasonably lower (greater) than the values of $P\left(\nu_{\mu} \rightarrow \nu_{e}\right)\left(P\left(\bar{\nu}_{\mu} \rightarrow \bar{\nu}_{e}\right)\right)$ for NH and any value of $\delta_{\mathrm{CP}}$. Hence, for these favourable combinations, $\mathrm{NO} \nu \mathrm{A}$ is capable of determining the hierarchy at a confidence level (C.L.) of $2 \sigma$ or better, with 3 years each of $\nu$ and $\bar{\nu}$ runs. However, as mentioned above, the change in the first term can be canceled by the second term for unfavourable values of $\delta_{\mathrm{CP}}$. This leads to hierarchy- $\delta_{\mathrm{CP}}$ degeneracy [2123]. From figure 1, we see that, $P\left(\nu_{\mu} \rightarrow \nu_{e}\right)$ and $P\left(\bar{\nu}_{\mu} \rightarrow \bar{\nu}_{e}\right)$ for NH and $\delta_{\mathrm{CP}}$ in the UHP are very close to or degenerate with those of IH and $\delta_{\mathrm{CP}}$ in the LHP. For these unfavourable combinations, $\mathrm{NO} \nu \mathrm{A}$ has no hierarchy sensitivity [15]. Addition of $\mathrm{T} 2 \mathrm{~K}$ data gives rise to a small sensitivity $[23,24]$. In this paper, we explore the further degeneracies in the case of the favourable hierarchy- $\delta_{\mathrm{CP}}$ combinations.

\section{$2.2 \theta_{13}$-hierarchy degeneracy}

Even if $\delta_{\mathrm{CP}}$ is in the favourable half-plane, there are further degeneracies which limit the hierarchy sensitivity of an experiment. For example, in eq. (2.1), the increase (reduction) 
in the first term for $\mathrm{NH}$ (IH) case, due to matter effect, can be canceled by choosing a lower (higher) value of $\theta_{13}$. This $\theta_{13}$-hierarchy degeneracy [21] can reduce the hierarchy sensitivity. However, a combination of $\nu$ and $\bar{\nu}$ data is not susceptible to this degeneracy. The reason is the following. In $\nu$ data, it is possible to have $P\left(\nu_{\mu} \rightarrow \nu_{e}\right)\left(\theta_{13}, N H\right) \approx$ $P\left(\nu_{\mu} \rightarrow \nu_{e}\right)\left(\theta_{13}{ }^{\prime}, I H\right)$ with $\theta_{13}{ }^{\prime}>\theta_{13}$. However, for such a choice of $\theta_{13}{ }^{\prime}$, we will have $P\left(\bar{\nu}_{\mu} \rightarrow \bar{\nu}_{e}\right)\left(\theta_{13}, N H\right)$ significantly smaller than $P\left(\bar{\nu}_{\mu} \rightarrow \bar{\nu}_{e}\right)\left(\theta_{13}{ }^{\prime}, I H\right)$. Thus a degeneracy in the $\nu$ data is resolved by the $\bar{\nu}$ data (and vice-verse). If the allowed range of $\theta_{13}$ is large, then a combination of $\nu$ and $\bar{\nu}$ data has better hierarchy sensitivity compared to pure $\nu$ data.

\subsection{Octant-hierarchy degeneracy}

A more serious degeneracy, which limits the hierarchy sensitivity, is the octant-hierarchy degeneracy. MINOS experiment has measured $\sin ^{2} 2 \theta_{23}<1[8]$ and the global fits favour a non-maximal value of $\theta_{23}$ [9-11]. There are two degenerate solutions, with $\theta_{23}$ in the lower octant (LO) $\left(\sin ^{2} \theta_{23}<0.5\right)$ and with $\theta_{23}$ in the higher octant $(\mathrm{HO})\left(\sin ^{2} \theta_{23}>0.5\right)$. Thus we have four possible octant-hierarchy combinations: LO-NH, HO-NH, LO-IH and HO-IH. As already stated, the first term in $P\left(\nu_{\mu} \rightarrow \nu_{e}\right)$ becomes larger (smaller) for $\mathrm{NH}(\mathrm{IH})$. The same term also becomes smaller (larger) for LO (HO). If the case HO-NH (LO-IH) is true, then the values of $P\left(\nu_{\mu} \rightarrow \nu_{e}\right)$ are significantly higher (smaller) than those for IH (NH) and any octant. For these two cases, pure $\nu$ data has good hierarchy determination capability. But the situation is very different for the two cases LO-NH and HO-IH. The increase (decrease) in the first term of $P\left(\nu_{\mu} \rightarrow \nu_{e}\right)$ due to $\mathrm{NH}$ (IH) is canceled (compensated) by the choice of $\mathrm{LO}(\mathrm{HO})$. Thus the two cases, LO-NH and HO-IH, have degenerate values for $P\left(\nu_{\mu} \rightarrow \nu_{e}\right)$. However, this degeneracy is not present in $P\left(\bar{\nu}_{\mu} \rightarrow \bar{\nu}_{e}\right)$, which receives a double boost (suppression) for the case of HO-IH (LO-NH). Thus the octant-hierarchy degeneracy in $P\left(\nu_{\mu} \rightarrow \nu_{e}\right)$ is broken by $P\left(\bar{\nu}_{\mu} \rightarrow \bar{\nu}_{e}\right)$ (and vice-verse) as in the case of $\theta_{13}$-hierarchy degeneracy. Therefore pure $\nu$ data has no hierarchy sensitivity if the cases LO-NH or HO-IH are true, but a combination of $\nu$ and $\bar{\nu}$ data will have a good sensitivity.

\section{Results}

\subsection{Simulation details}

In this report, we study the possible hierarchy reach of the first three years of $\mathrm{NO} \nu \mathrm{A}$ data. As shown in the previous section, a pure $\nu$ data is subject to $\theta_{13}$-hierarchy and octanthierarchy degeneracies, whereas a combination of $\nu$ and $\bar{\nu}$ data is not. Therefore, here we consider two options: (a) a 3 year $\nu$ run (labeled $3 \nu$ in the rest of the paper) and (b) equal $\nu$ and $\bar{\nu}$ runs of 1.5 years each (labeled $1.5 \nu+1.5 \bar{\nu}$ ).

$\mathrm{NO} \nu \mathrm{A}$ experiment [25] consists of a 14 kiloton totally active scintillator detector (TASD), placed $810 \mathrm{~km}$ away from Fermilab, situated at a $0.8^{\circ}$ off-axis location from the NuMI beam. The $\nu$ flux peaks sharply at $2 \mathrm{GeV}$, close to the energy range $1.4-1.8 \mathrm{GeV}$, where the oscillation maxima occur for $\mathrm{NH}$ and for IH. It is scheduled to have equal $\nu$ and $\bar{\nu}$ runs of 3 years each, with a NuMI beam power of $700 \mathrm{~kW}$, corresponding to $6 \times 10^{20}$ 
protons on target per year. In our simulations, we have used the re-tuned signal acceptance and background rejection factors taken from [24, 26]. In the numerical simulations, we took the solar oscillation parameters to be $\sin ^{2} \theta_{12}=0.30$ and $\Delta_{21}=7.5 \times 10^{-5} \mathrm{eV}^{2}$, which have been kept fixed [11]. The other parameters used are $\sin ^{2} 2 \theta_{13}=0.089$ and $\Delta m_{\text {eff }}^{2}= \pm 2.4 \times 10^{-3} \mathrm{eV}^{2}$ [8], where the positive (negative) sign is for $\mathrm{NH}(\mathrm{IH}) . \Delta_{31}$ is derived from $\Delta m_{\text {eff }}^{2}$ from the expression given in [27]. For $\theta_{23}$, we considered the cases of both maximal and non-maximal mixing. For maximal mixing $(\mathrm{MM}), \sin ^{2} \theta_{23}=0.5$. For non-maximal mixing, we have used the two degenerate best-fit values of the global fits: 0.41 for $\theta_{23}$ in LO and 0.59 for $\theta_{23}$ in HO [11].

The spectrum of electron neutrino appearance events and that of the electron antineutrino appearance events are first computed for an assumed true hierarchy. The same quantities are calculated again for the wrong hierarchy and the $\Delta \chi^{2}$ is computed between the event spectra for the true and the wrong hierarchies. The event spectrum simulations and the $\Delta \chi^{2}$ calculation are done by using the software GLoBES [28, 29]. The minimum $\Delta \chi^{2}$ is computed by doing a marginalization over the neutrino parameters. We took $\sigma\left(\Delta m_{\text {eff }}^{2}\right)=3 \%$ [30] and $\sigma\left(\sin ^{2} 2 \theta_{13}\right)=10 \%$ in the preliminary calculations and $5 \%$ in later calculations. For both these parameters, the marginalization was done over $2 \sigma$ range with Gaussian priors. The marginalization range for $\sin ^{2} \theta_{23}$ is its $3 \sigma$ allowed range: $[0.35,0.65]$ and that of $\delta_{\mathrm{CP}}$ is the full range $\left[-180^{\circ}, 180^{\circ}\right] .^{1}$ No priors were added for these two parameters.

\subsection{Effect of precision of $\sin ^{2} 2 \theta_{13}$ on hierarchy determination}

In figure 2 we have shown the hierarchy determination potential of $\mathrm{NO} \nu \mathrm{A}$ assuming a $10 \%$ uncertainty in $\sin ^{2} 2 \theta_{13}$. The plots show $\Delta \chi^{2}$ vs. $\delta_{\mathrm{CP}}\left(\right.$ true) for $\theta_{23}=45^{\circ}$, for both $3 \nu$ and $1.5 \nu+1.5 \bar{\nu}$ runs. The left panel is for NH and LHP and the right panel is for IH and UHP. We see from these plots that a $2 \sigma$ hierarchy determination is possible for about $50 \%$ of the favourable half plane for $1.5 \nu+1.5 \bar{\nu}$ run, whereas a $3 \nu$ run can determine hierarchy for only a smaller range. In particular, if IH and UHP is true, a $2 \sigma$ hierarchy determination is not possible for any $\delta_{\mathrm{CP}}$. Here the number of $\sigma$ is taken to be $\sqrt{\Delta \chi^{2}}$.

The lower sensitivity of $3 \nu$ run is due to the marginalization over $\theta_{13}$. Because of the relatively large range of variation for $\theta_{13}{ }^{\prime}$, it is possible for $P\left(\nu_{\mu} \rightarrow \nu_{e}\right)\left(\theta_{13}{ }^{\prime}, I H\right)$ to come reasonably close to $P\left(\nu_{\mu} \rightarrow \nu_{e}\right)\left(\theta_{13}, N H\right)$, thus reducing the $\Delta \chi^{2}$. As explained in the previous section, the $1.5 \nu+1.5 \bar{\nu}$ run is less sensitive to this marginalization and gives a larger $\Delta \chi^{2}$. If the uncertainty in $\sin ^{2} 2 \theta_{13}$ is reduced to $5 \%$. the hierarchy reach for $3 \nu$ does improve and becomes equal to that of $1.5 \nu+1.5 \bar{\nu}$ run.

\subsection{Resolving the octant-hierarchy degeneracy}

We now assume that $\sigma\left(\sin ^{2} 2 \theta_{13}\right)=5 \%$ and take $\theta_{23}$ to be non-maximal. Once again we limit ourselves to the favourable hierarchy- $\delta_{\mathrm{CP}}$ combinations, NH and LHP and IH and UHP. But, because of the octant degeneracy of $\theta_{23}$, we must consider four possible combinations of octant and hierarchy: LO-NH, HO-NH, LO-IH and HO-IH.

\footnotetext{
${ }^{1}$ The global best fit $[31,32]$ indicates a preference for $\delta_{\mathrm{CP}}$ to be in the LHP. But here, we will be conservative and consider the full range of $\delta_{\mathrm{CP}}$ in our marginalization.
} 


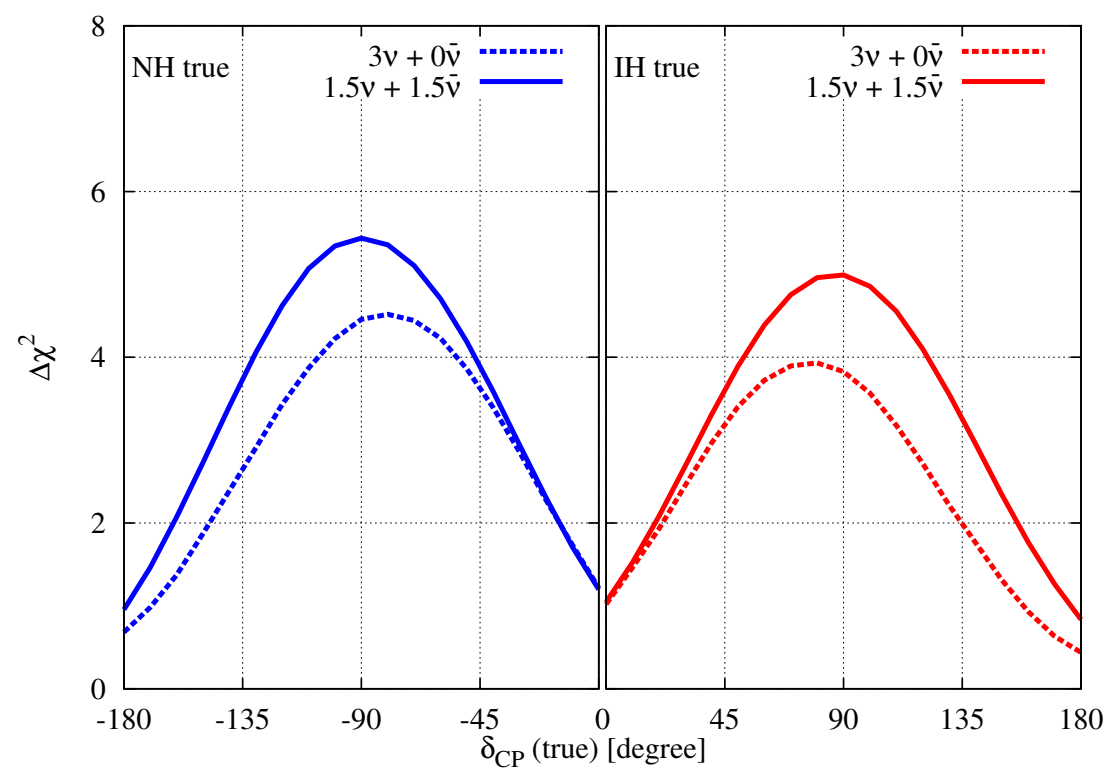

Figure 2. Hierarchy sensitivity assuming $10 \%$ uncertainty in $\sin ^{2} 2 \theta_{13}$ and maximal $\theta_{23}$. In the left (right) panel, the true hierarchy is taken to be $\mathrm{NH}(\mathrm{IH})$.

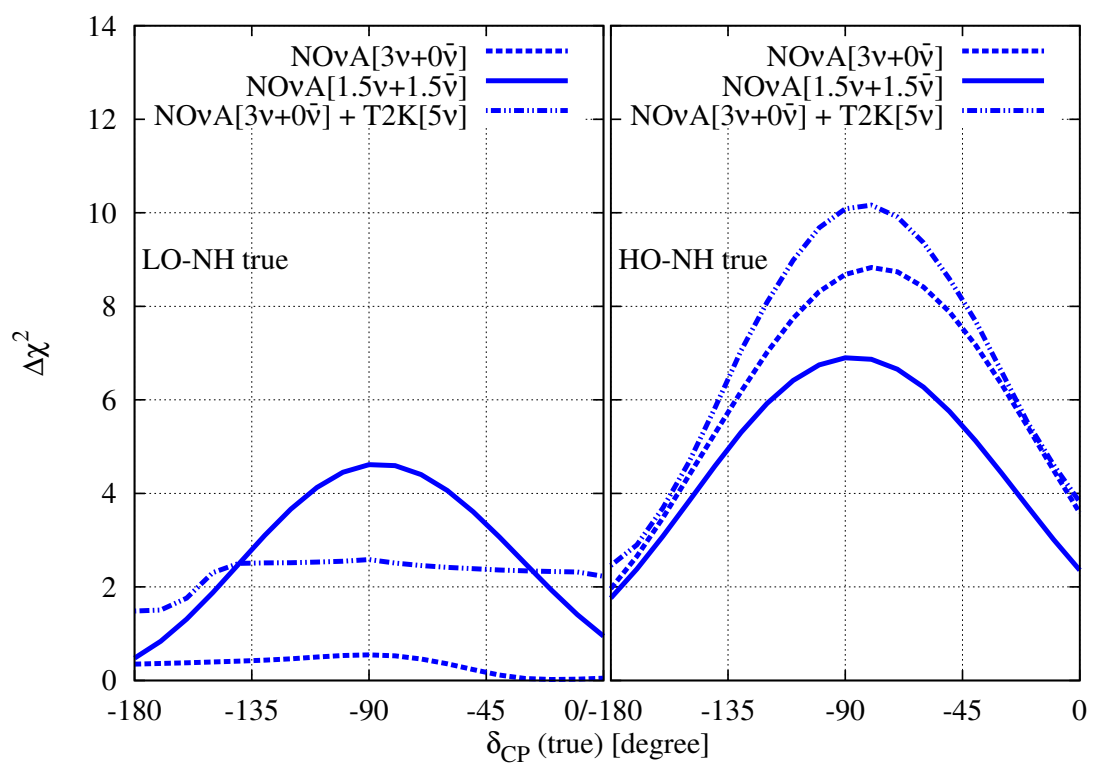

Figure 3. Hierarchy sensitivity assuming $5 \%$ uncertainty in $\sin ^{2} 2 \theta_{13}$ for NH and LHP. In the left (right) panel, the true $\sin ^{2} \theta_{23}$ is taken to be $0.41(0.59)$.

In figure 3, we show the hierarchy capability assuming NH and LHP. The left (right) panel corresponds to $\theta_{23}$ in $\mathrm{LO}(\mathrm{HO})$. In figure 4, we do the same for IH and UHP. From these figures, we see that for HO-NH and LO-IH, $3 \nu$ run does have a better hierarchy reach compared to $1.5 \nu+1.5 \bar{\nu}$ run and is capable of giving a better than $2 \sigma$ hierarchy discrimination for more than half of the favourable half plane. But, for the other two possibilities, LO-NH and HO-IH, $3 \nu$ run has no hierarchy sensitivity whereas $1.5 \nu+1.5 \bar{\nu}$ 


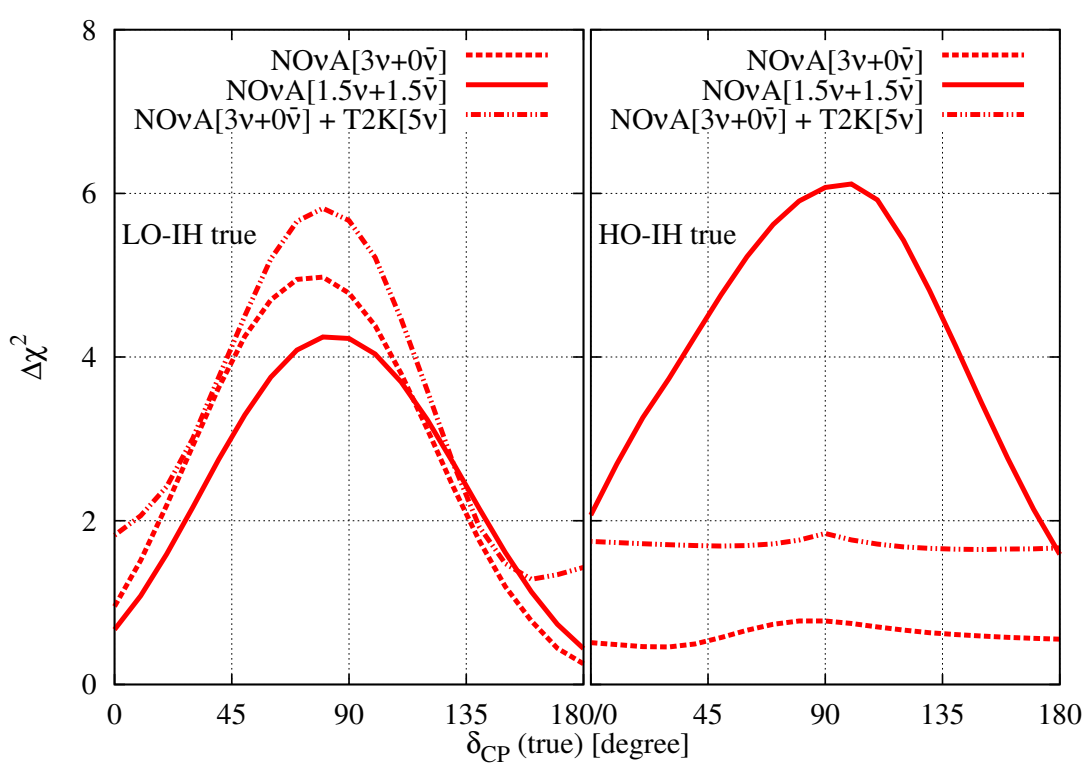

Figure 4. Hierarchy sensitivity assuming $5 \%$ uncertainty in $\sin ^{2} 2 \theta_{13}$ for IH and UHP. In the left (right) panel, the true $\sin ^{2} \theta_{23}$ is taken to be $0.41(0.59)$.

run has reasonable hierarchy sensitivity. The very small values of $\Delta \chi^{2}$, for the $3 \nu$ run, occur due to the marginalization over $\sin ^{2} \theta_{23}$ and $\delta_{\mathrm{CP}}$. Addition of 5 year $\nu$ data from T2K leads only to a small improvement.

As mentioned before, the dominant term in $P\left(\nu_{\mu} \rightarrow \nu_{e}\right)$ is proportional to $\sin ^{2} 2 \theta_{13} \sin ^{2} \theta_{23}$. Matter effects in NH make this term larger and choosing HO makes it even larger. Hence, for $\delta_{\mathrm{CP}}$ in LHP, $P\left(\nu_{\mu} \rightarrow \nu_{e}\right)(\mathrm{HO}-\mathrm{NH})$ is significantly higher than $P\left(\nu_{\mu} \rightarrow \nu_{e}\right)(\mathrm{IH})$ for any values of neutrino parameters. Because of the double increase in the probability, the statistics for $\mathrm{HO}-\mathrm{NH}$ will be quite large. Hence, this combination has $2 \sigma$ hierarchy discrimination for $87 \%(68 \%)$ of the favourable half-plane for $3 \nu(1.5 \nu+1.5 \bar{\nu})$ run. Matter effects in IH make the leading term in $P\left(\nu_{\mu} \rightarrow \nu_{e}\right)$ smaller and choosing LO makes it even smaller. So, for $\delta_{\mathrm{CP}}$ in UHP, $P\left(\nu_{\mu} \rightarrow \nu_{e}\right)$ (LO-IH) is significantly smaller than $P\left(\nu_{\mu} \rightarrow \nu_{e}\right)(\mathrm{NH})$ for any values of neutrino parameters. This double decrease in probability, leads to the lowest statistics for LO-IH. Here, $3 \nu(1.5 \nu+1.5 \bar{\nu})$ run can determine hierarchy at $2 \sigma$ for $35 \%(20 \%)$ of favourable half-plane. However, it must be emphasized that, in these two cases HO-NH and LO-IH, the hierarchy reach of $1.5 \nu+1.5 \bar{\nu}$ is only slightly worse than that of $3 \nu$.

But, for the combination of LO-NH, the choice of NH increases $P\left(\nu_{\mu} \rightarrow \nu_{e}\right)$ whereas the choice of LO lowers it. Similarly, for the combination HO-IH, the choice of IH lowers $P\left(\nu_{\mu} \rightarrow \nu_{e}\right)$ and the choice of $\mathrm{HO}$ increases it. The marginalization over $\theta_{23}$ and $\delta_{\mathrm{CP}}$ leads to a wrong hierarchy probability being very close to the true hierarchy probability. Thus, it is possible to have $P\left(\nu_{\mu} \rightarrow \nu_{e}\right)\left(\mathrm{NH}, \theta_{23}<45^{\circ}, \delta_{\mathrm{CP}}\right)$ mimic $P\left(\nu_{\mu} \rightarrow \nu_{e}\right)\left(\mathrm{IH}, \theta_{23}{ }^{\prime}>45^{\circ}\right.$, $\delta_{\mathrm{CP}}^{\prime}$ ), where $\theta_{23}$ and $\theta_{23}{ }^{\prime}$ may or may not be complementary and $\delta_{\mathrm{CP}}$ and $\delta_{\mathrm{CP}}^{\prime}$ may or may not be equal. But, in the case of $\bar{\nu}$, both the choices $\mathrm{LO}$ and $\mathrm{NH}$ lead to a reduction in the probability and both the choices $\mathrm{HO}$ and IH increase the probability. Whenever it is 


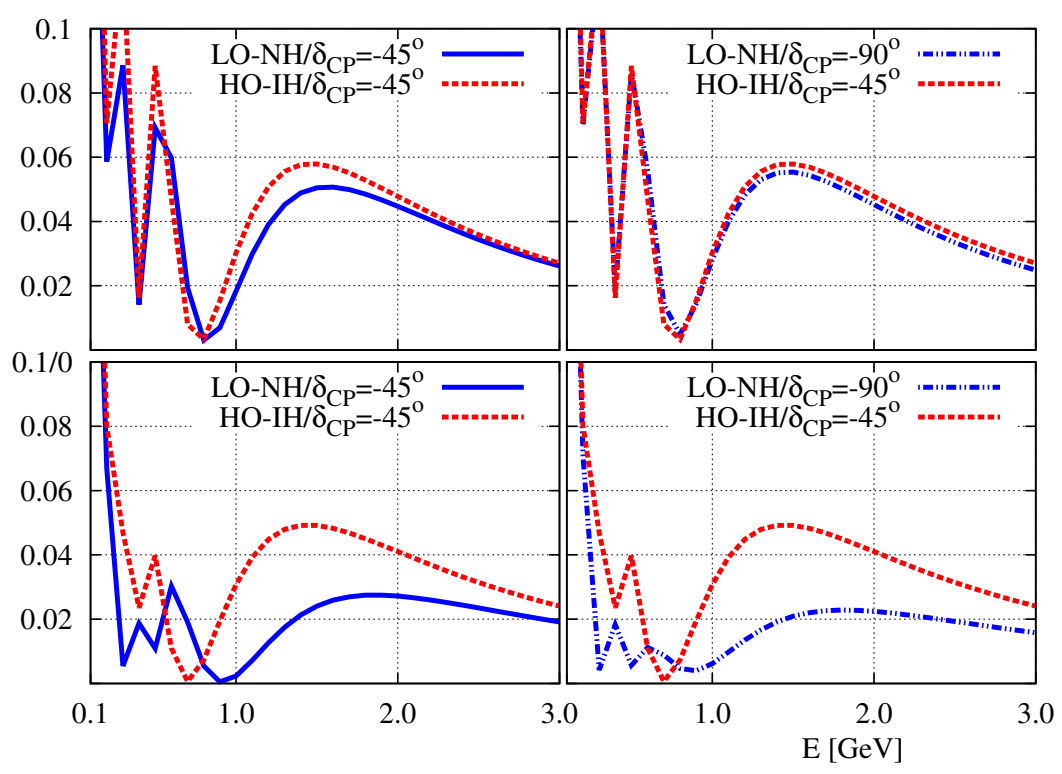

Figure 5. Illustration of degenerate $P\left(\nu_{\mu} \rightarrow \nu_{e}\right)$ and non-degenerate $P\left(\bar{\nu}_{\mu} \rightarrow \bar{\nu}_{e}\right)$ for the following two cases. Left: $\left(\mathrm{LO}-\mathrm{NH}, \delta_{\mathrm{CP}}=-45^{\circ}\right)$ and $\left(\mathrm{HO}-\mathrm{IH}, \delta_{\mathrm{CP}}^{\prime}=-45^{\circ}\right)$, Right: $\left(\mathrm{LO}-\mathrm{NH}, \delta_{\mathrm{CP}}=-90^{\circ}\right)$ and $\left(\mathrm{HO}-\mathrm{IH}, \delta_{\mathrm{CP}}^{\prime}=-45^{\circ}\right)$.

possible to have $P\left(\nu_{\mu} \rightarrow \nu_{e}\right)\left(\mathrm{NH}, \theta_{23}, \delta_{\mathrm{CP}}\right) \approx P\left(\nu_{\mu} \rightarrow \nu_{e}\right)\left(\mathrm{IH}, \theta_{23}{ }^{\prime}, \delta_{\mathrm{CP}}{ }^{\prime}\right)$, the corresponding values of $P\left(\bar{\nu}_{\mu} \rightarrow \bar{\nu}_{e}\right)$ will be far apart. This is illustrated in figure 5 for two cases, where $\theta_{23}$ and $\theta_{23}{ }^{\prime}$ are complementary. For the two left panels $\delta_{\mathrm{CP}}=\delta_{\mathrm{CP}}^{\prime}$ and for the two right panels $\delta_{\mathrm{CP}} \neq \delta_{\mathrm{CP}}^{\prime}$. The large separation in $P\left(\bar{\nu}_{\mu} \rightarrow \bar{\nu}_{e}\right)$ leads to a far better hierarchy discrimination for $1.5 \nu+1.5 \bar{\nu}$ run compared to $3 \nu$ run. All the results discussed above are neatly summarized in the table I. In all cases, the $1.5 \nu+1.5 \bar{\nu}$ data is insensitive to the uncertainty in $\sin ^{2} 2 \theta_{13}$. Except for the no-sensitivity combinations, LO-NH and HO-IH, the $3 \nu$ data shows noticeable improvement when the uncertainty is reduced to $5 \%$ but none with further reduction to $2 \%$.

In the most recent global fits of the neutrino oscillation data [31], the best-fit value of $\sin ^{2} \theta_{23}$ in LO is $0.45,(i$. e e closer to the maximal mixing value), though the best-fit value in $\mathrm{HO}$ remains at 0.59 . We have redone our calculations and compared the hierarchy discrimination ability of $3 \nu$ vs $1.5 \nu+1.5 \bar{\nu}$ data of $\mathrm{NO} \nu \mathrm{A}$, for these new values of $\sin ^{2} \theta_{23}$. These results are shown in figures 6 and 7 . As we see from these figures, even with the smaller deviation of $\theta_{23}$ from maximality, the $3 \nu$ run of $\mathrm{NO} \nu \mathrm{A}$ has no hierarchy sensitivity for the two combinations LO-NH and $\mathrm{HO}-\mathrm{IH}$, whereas the $1.5 \nu+1.5 \bar{\nu}$ run has good hierarchy determination capability for all four combinations.

The most recent results of the T2K experiment [33] give $\sin ^{2} \theta_{23}=0.514_{-0.055}^{+0.055}(0.511 \pm$ $0.055)$ for $\mathrm{NH}(\mathrm{IH})$. These values seem to favour maximal mixing but a deviation from maximality is also very likely. The parameters we have chosen here fall within the $2 \sigma$ range of these measurements. Even if the deviation of $\theta_{23}$ from maximality is very small $\left(\left|\sin ^{2} \theta_{23}-0.5\right|=0.02\right)$, the hierarchy sensitivity of $1.5 \nu+1.5 \bar{\nu}$ run is better than that of $3 \nu$ run for the two combinations LO-NH and HO-IH. This is illustrated in figures 8 and 9. 


\begin{tabular}{|c|ccc|ccc|ccc|}
\hline$\delta_{\mathrm{CP}}:$ & \multicolumn{3}{|c|}{$-180^{\circ}$} & \multicolumn{3}{c|}{$-135^{\circ}$} & \multicolumn{3}{c|}{$-90^{\circ}$} \\
\cline { 2 - 10 } $\sin ^{2} \theta_{23}:$ & 0.41 & 0.5 & 0.59 & 0.41 & 0.5 & 0.59 & 0.41 & 0.5 & 0.59 \\
\hline \multirow{3}{*}{$1.5 \nu+1.5 \bar{\nu}$} & 0.47 & 0.97 & 1.76 & 2.80 & 3.76 & 4.91 & 4.52 & 5.52 & 6.71 \\
& 0.47 & 0.97 & 1.76 & 2.80 & 3.76 & 4.95 & 4.61 & 5.53 & 6.93 \\
& 0.47 & 0.97 & 1.76 & 2.80 & 3.76 & 4.95 & 4.61 & 5.53 & 6.96 \\
\hline \multirow{3}{*}{$3 \nu+0 \bar{\nu}$} & 0.56 & 0.75 & 1.66 & 1.10 & 2.90 & 4.61 & 1.23 & 4.65 & 6.89 \\
& 0.56 & 0.75 & 1.98 & 1.10 & 3.37 & 5.76 & 1.23 & 5.65 & 8.68 \\
& 0.56 & 0.75 & 2.10 & 1.10 & 3.61 & 6.21 & 1.23 & 6.06 & 9.45 \\
\hline
\end{tabular}

\begin{tabular}{|c|ccc|ccc|ccc|}
\hline $\begin{array}{c}\delta_{\mathrm{CP}}: \\
\sin ^{2} \theta_{23}:\end{array}$ & \multicolumn{4}{|c|}{0} & \multicolumn{3}{|c|}{$45^{\circ}$} & \multicolumn{3}{c|}{$90^{\circ}$} \\
\cline { 2 - 10 } & 0.41 & 0.5 & 0.59 & 0.41 & 0.5 & 0.59 & 0.41 & 0.5 & 0.59 \\
\hline \multirow{3}{*}{$1.5 \nu+1.5 \bar{\nu}$} & 0.67 & 1.10 & 1.92 & 2.91 & 3.70 & 4.37 & 4.17 & 5.08 & 5.89 \\
& 0.67 & 1.10 & 2.10 & 3.03 & 3.81 & 4.53 & 4.22 & 5.16 & 6.11 \\
& 0.67 & 1.10 & 2.10 & 3.03 & 3.81 & 4.53 & 4.22 & 5.16 & 6.12 \\
\hline \multirow{3}{*}{$3 \nu+0 \bar{\nu}$} & 0.74 & 1.07 & 0.51 & 3.10 & 3.20 & 0.53 & 3.78 & 3.81 & 0.77 \\
& 0.99 & 1.41 & 0.51 & 3.92 & 4.05 & 0.53 & 4.77 & 4.83 & 0.77 \\
& 1.02 & 1.52 & 0.51 & 4.28 & 4.43 & 0.53 & 5.16 & 5.23 & 0.77 \\
\hline
\end{tabular}

Table 1. Hierarchy discrimination reach of $\mathrm{NO} \nu \mathrm{A}$ data for $1.5 \nu+1.5 \bar{\nu}$ and $3 \nu$ runs. The upper (lower) half is for NH-LHP (IH-UHP) true. In each case, the $\Delta \chi^{2}$ values are shown for $\theta_{23}$ being in LO, maximal and in $\mathrm{HO}$ and for three values of $\delta_{\mathrm{CP}}$, covering half of the favourable half plane. $\Delta \chi^{2}$ values for the other half are nearly symmetric about $\delta_{\mathrm{CP}}= \pm 90^{\circ}$, as can be seen from the figures. 3 and 4 . The three lines in each small box correspond to $10 \%, 5 \%$ and $2 \%$ precision in $\sin ^{2} 2 \theta_{13}$ respectively.



Figure 6. Hierarchy sensitivity assuming $5 \%$ uncertainty in $\sin ^{2} 2 \theta_{13}$ for NH and LHP. In the left (right) panel, the true $\sin ^{2} \theta_{23}$ is taken to be $0.45(0.59)$. 


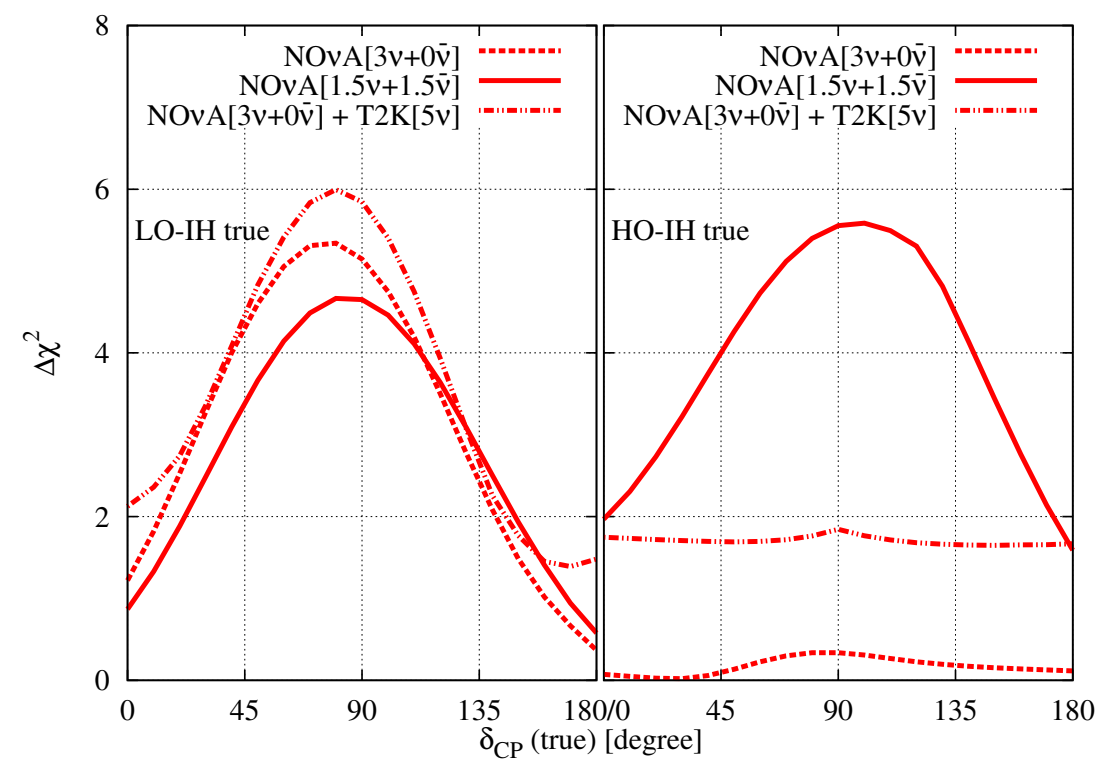

Figure 7. Hierarchy sensitivity assuming $5 \%$ uncertainty in $\sin ^{2} 2 \theta_{13}$ for IH and UHP. In the left (right) panel, the true $\sin ^{2} \theta_{23}$ is taken to be $0.45(0.59)$.

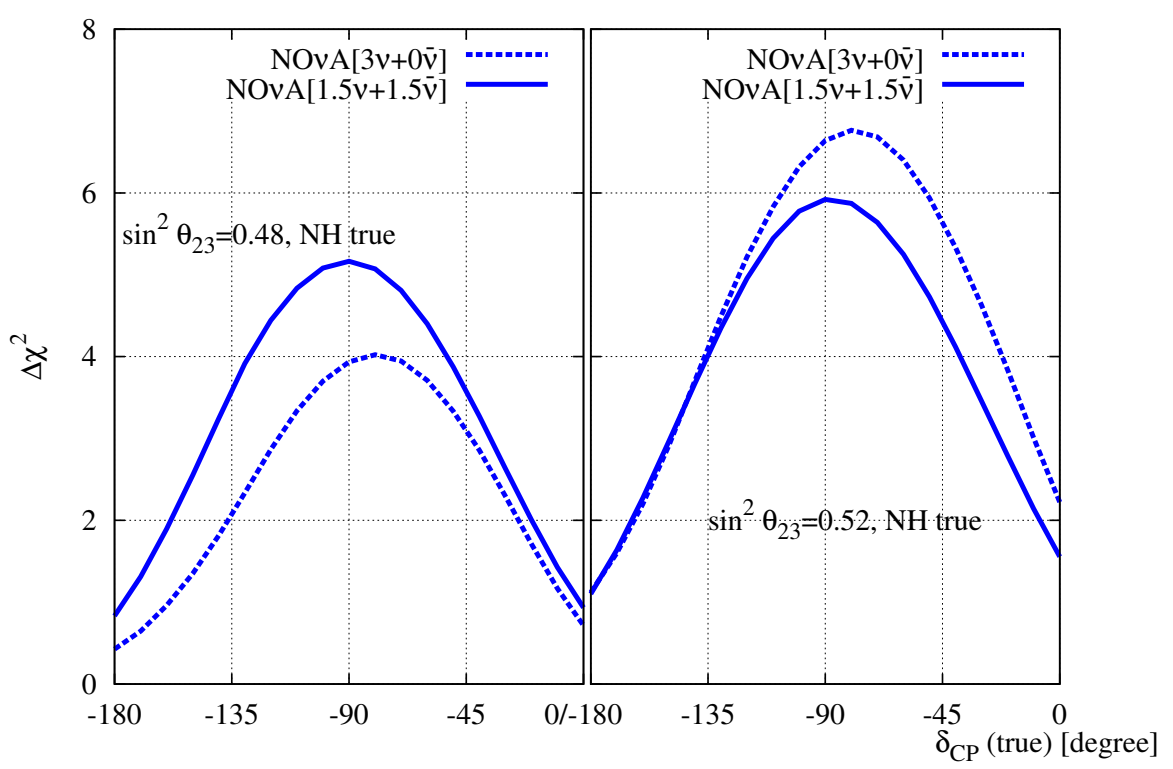

Figure 8. Hierarchy sensitivity assuming $5 \%$ uncertainty in $\sin ^{2} 2 \theta_{13}$ for NH and LHP. In the left (right) panel, the true $\sin ^{2} \theta_{23}$ is taken to be $0.48(0.52)$.

\subsection{Advantages of $1.5 \nu+1.5 \bar{\nu}$ run of $\mathrm{NO} \nu \mathrm{A}$}

In the previous subsection, we have argued that the $1.5 \nu+1.5 \bar{\nu}$ run of $\mathrm{NO} \nu \mathrm{A}$ has good hierarchy sensitivity for all four combinations of octant and hierarchy whereas the $3 \nu$ run has a slightly better hierarchy sensitivity for the two combinations HO-NH and LO-IH. Thus it becomes important to address the question: can 1.5 years of $\nu$ data of $\mathrm{NO} \nu \mathrm{A}$ give 


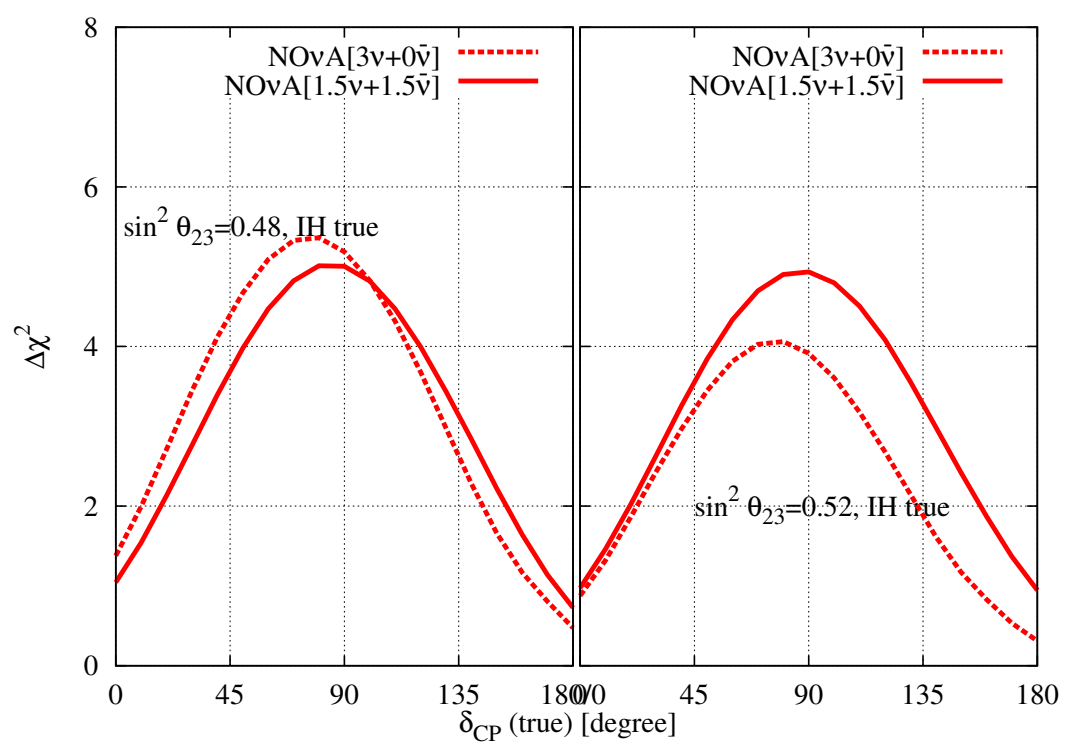

Figure 9. Hierarchy sensitivity assuming $5 \%$ uncertainty in $\sin ^{2} 2 \theta_{13}$ for IH and UHP. In the left (right) panel, the true $\sin ^{2} \theta_{23}$ is taken to be $0.48(0.52)$.

a hint of hierarchy if either HO-NH or LO-IH are the true combinations? Based on the results of the previous sub-section, we know that there will be no sensitivity if LO-NH or HO-IH are true. For the other two cases, HO-NH and LO-IH, the hierarchy sensitivity from the $1.5 \nu$ data is given in figure 10. From this figure, we see that there is reasonable hierarchy sensitivity for the combination HO-NH, even from 1.5 years of $\nu$ data, but not for the combination LO-IH. This is expected because $P\left(\nu_{\mu} \rightarrow \nu_{e}\right)$ receives a double boost in the case of HO-NH and hence there will be a large number of signal events. For LO-IH, $P\left(\nu_{\mu} \rightarrow \nu_{e}\right)$ gets a double suppression and hence the statistics in the $1.5 \nu$ run are not sufficient to rule out the wrong hierarchy. Addition of 2 years of $\nu$ data from T2K leads to no significant change.

This leads us to a very interesting conclusion: the physics capabilities of $\mathrm{NO} \nu \mathrm{A}$ are enhanced if it has $1.5 \nu+1.5 \bar{\nu}$ runs during the first three years. This statement is true for any octant-hierarchy combination. We see above that, for the combination of HO-NH, a $2 \sigma$ hint of hierarchy is possible for half of LHP, even with 1.5 years of $\nu$ run. If the hierarchy is known after such a run, then a run plan, which has the best CP sensitivity, is preferable. To maximize the CP sensitivity, it is desirable to have equal number of $\nu$ and $\bar{\nu}$ events [34]. This requires a longer $\bar{\nu}$ run because the $\bar{\nu}$ cross sections are smaller. Hence, if HO-NH is true, a hierarchy hint can be obtained with a $1.5 \nu$ run, after which it is preferable to run $\mathrm{NO} \nu \mathrm{A}$ in $\bar{\nu}$ mode only. For the other three octant-hierarchy combinations, $1.5 \nu$ run does not give a hint of hierarchy. In such a situation, a switch to $\bar{\nu}$ run will guarantee a $2 \sigma$ hierarchy discrimination for a reasonable fraction of the favourable half plane of $\delta_{\mathrm{CP}}$.

\section{Conclusions}

$\mathrm{NO} \nu \mathrm{A}$ experiment is about to start taking data. Among its physics goals are (a) the determination of neutrino mass hierarchy, (b) the determination of the octant of $\theta_{23}$ and 


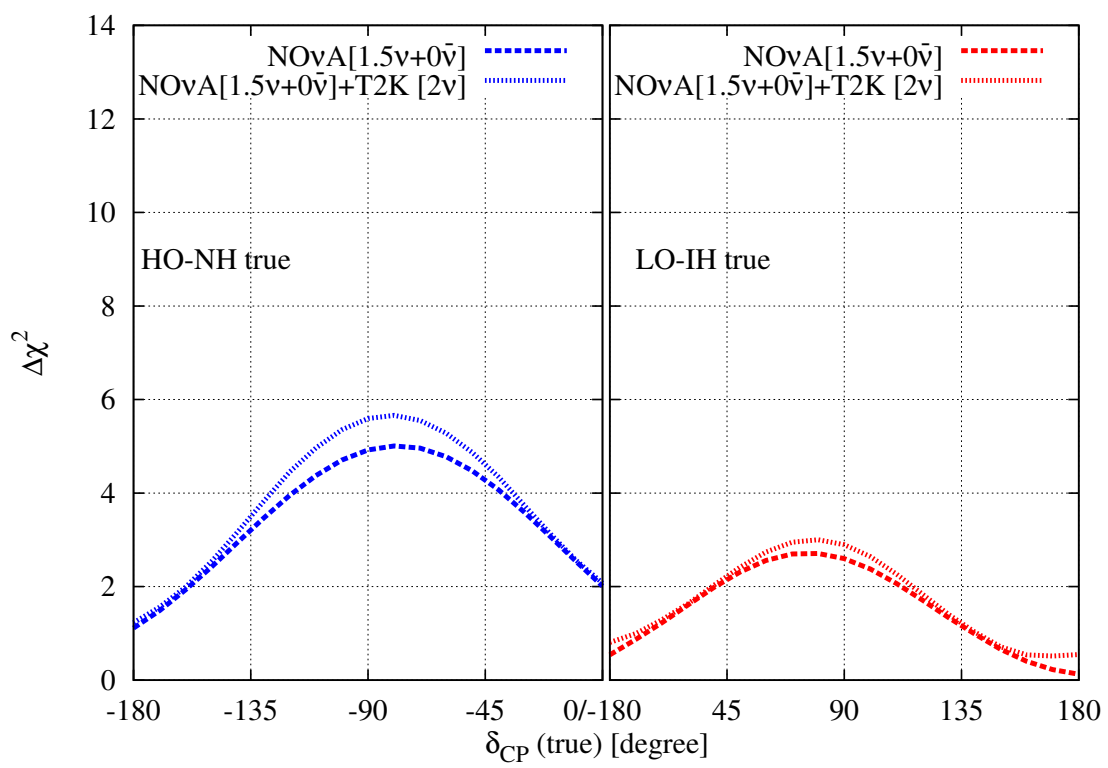

Figure 10. Hierarchy sensitivity of 1.5 years of $\nu$ run for HO-NH (left panel) and LO-IH (right panel). In the left (right) panel, the true $\sin ^{2} \theta_{23}$ is taken to be $0.59(0.41)$.

(c) the discovery of leptonic $\mathrm{CP}$ violation. The hierarchy reach of pure $\nu$ data is subject to $\theta_{13}$-hierarchy and octant-hierarchy degeneracies, whereas equal $\nu-\bar{\nu}$ runs are free from them. If the uncertainty in $\sin ^{2} 2 \theta_{13}$ remains at the present $10 \%$ level, then the combination $1.5 \nu+1.5 \bar{\nu}$ run has better hierarchy sensitivity compared to pure $3 \nu$ run. Even when this uncertainty is reduced to $5 \%$, the $3 \nu$ run fails to give any hierarchy discrimination, if the true combinations are LO-NH or HO-IH, whereas the combined $1.5 \nu+1.5 \bar{\nu}$ run has good hierarchy discrimination for all four octant-hierarchy combinations.

We argue that it is advantageous for $\mathrm{NO} \nu \mathrm{A}$ to have equal 1.5 years of $\nu$ and $\bar{\nu}$ runs during the first three years. We find that $1.5 \nu$ run gives a $2 \sigma$ hierarchy hint if the combination HO-NH is true and $\delta_{\mathrm{CP}}$ is in LHP. In such a situation, it is better to switch to $\bar{\nu}$ to maximize the $\mathrm{CP}$ sensitivity. For the other three octant-hierarchy combinations, $1.5 \nu$ run has poor or no hierarchy sensitivity. Following this up with a 1.5 year $\bar{\nu}$ run will give a better chance of hierarchy discrimination, if $\delta_{\mathrm{CP}}$ is in the favourable half plane.

Finally, what should happen after $1.5 \nu+1.5 \bar{\nu}$ run? If no hint of hierarchy is obtained, then a farther $1.5 \nu+1.5 \bar{\nu}$ run seems preferable. Then, the full hierarchy discrimination capability of $3 \nu+3 \bar{\nu}$ run of $\mathrm{NO} \nu \mathrm{A}$ will be realised. If a hint of hierarchy is found, then having the additional run in $\bar{\nu}$ mode is likely to give the best $\mathrm{CP}$ sensitivity.

\section{Acknowledgments}

We thank Sanjib Agarwalla for discussions on implementing re-tuned $\mathrm{NO} \nu \mathrm{A}$ efficiencies in GLoBES. We thank Maury Goodman, Mark Messier and Jon Urheim for their comments on the manuscript. S. U. S. thanks Stephen Parke, Peter Shanahan and other members of $\mathrm{NO} \nu \mathrm{A}$ group at Fermilab and Carlos Wagner, Maury Goodman and other members of 
$\mathrm{NO} \nu \mathrm{A}$ group at Argonne for their hospitality and for discussions related to this paper. We thank Srubabati Goswami for a critical reading of the manuscript. U. R. thanks Council for Scientific and Industrial Research (CSIR), Government of India, for financial support.

Open Access. This article is distributed under the terms of the Creative Commons Attribution License (CC-BY 4.0), which permits any use, distribution and reproduction in any medium, provided the original author(s) and source are credited.

\section{References}

[1] DAYA-BAY collaboration, F.P. An et al., Observation of electron-antineutrino disappearance at Daya Bay, Phys. Rev. Lett. 108 (2012) 171803 [arXiv:1203.1669] [INSPIRE].

[2] RENO collaboration, J.K. Ahn et al., Observation of Reactor Electron Antineutrino Disappearance in the RENO experiment, Phys. Rev. Lett. 108 (2012) 191802 [arXiv: 1204.0626] [INSPIRE].

[3] Double CHOOZ collaboration, Y. Abe et al., Reactor electron antineutrino disappearance in the Double CHOOZ experiment, Phys. Rev. D 86 (2012) 052008 [arXiv:1207.6632] [INSPIRE].

[4] CHOOZ collaboration, M. Apollonio et al., Initial results from the CHOOZ long baseline reactor neutrino oscillation experiment, Phys. Lett. B 420 (1998) 397 [hep-ex/9711002] [INSPIRE].

[5] CHOOZ collaboration, M. Apollonio et al., Limits on neutrino oscillations from the CHOOZ experiment, Phys. Lett. B 466 (1999) 415 [hep-ex/9907037] [INSPIRE].

[6] M. Narayan, G. Rajasekaran and S.U. Sankar, Three flavor implications of CHOOZ result, Phys. Rev. D 58 (1998) 031301 [hep-ph/9712409] [InSPIRE].

[7] Daya Bay Collaboration collaboration, D. Dwyer, Improved measurement of electron-5antineutrino disappearance at Daya Bay, Chin. Phys. C 37 (2013) 011001 [arXiv:1210.6327] [arXiv:1210.6327].

[8] MINOS collaboration, R. Nichol, Final MINOS results, (2012).

[9] D.V. Forero, M. Tortola and J.W.F. Valle, Global status of neutrino oscillation parameters after Neutrino-2012, Phys. Rev. D 86 (2012) 073012 [arXiv:1205.4018] [INSPIRE].

[10] G.L. Fogli et al., Global analysis of neutrino masses, mixings and phases: entering the era of leptonic CP-violation searches, Phys. Rev. D 86 (2012) 013012 [arXiv:1205.5254] [INSPIRE].

[11] M.C. Gonzalez-Garcia, M. Maltoni, J. Salvado and T. Schwetz, Global fit to three neutrino mixing: critical look at present precision, JHEP 12 (2012) 123 [arXiv:1209.3023] [INSPIRE].

[12] C.H. Albright, Normal vs. inverted hierarchy in type-I seesaw models, Phys. Lett. B 599 (2004) 285 [hep-ph/0407155] [INSPIRE].

[13] M. Fukugita and T. Yanagida, Baryogenesis without grand unification, Phys. Lett. B 174 (1986) 45 [INSPIRE].

[14] P. Huber, M. Lindner, T. Schwetz and W. Winter, First hint for CP-violation in neutrino oscillations from upcoming superbeam and reactor experiments, JHEP 11 (2009) 044 [arXiv:0907.1896] [INSPIRE].

[15] S. Prakash, S.K. Raut and S.U. Sankar, Getting the best out of T2K and NOvA, Phys. Rev. D 86 (2012) 033012 [arXiv:1201.6485] [INSPIRE]. 
[16] S.K. Agarwalla, S. Prakash and S.U. Sankar, Resolving the octant of $\theta_{23}$ with T2K and NOvA, JHEP 07 (2013) 131 [arXiv: 1301.2574] [INSPIRE].

[17] A. Chatterjee, P. Ghoshal, S. Goswami and S.K. Raut, Octant sensitivity for large $\theta_{13}$ in atmospheric and long baseline neutrino experiments, JHEP 06 (2013) 010 [arXiv: 1302.1370] [INSPIRE].

[18] A. Cervera et al., Golden measurements at a neutrino factory, Nucl. Phys. B 579 (2000) 17 [Erratum ibid. B 593 (2001) 731-732] [hep-ph/0002108] [INSPIRE].

[19] M. Freund, Analytic approximations for three neutrino oscillation parameters and probabilities in matter, Phys. Rev. D 64 (2001) 053003 [hep-ph/0103300] [INSPIRE].

[20] L. Wolfenstein, Neutrino oscillations in matter, Phys. Rev. D 17 (1978) 2369 [INSPIRE].

[21] V. Barger, D. Marfatia and K. Whisnant, Breaking eight fold degeneracies in neutrino CP-violation, mixing and mass hierarchy, Phys. Rev. D 65 (2002) 073023 [hep-ph/0112119] [INSPIRE].

[22] H. Minakata and H. Sugiyama, Exploring leptonic CP-violation by reactor and neutrino superbeam experiments, Phys. Lett. B 580 (2004) 216 [hep-ph/0309323] [INSPIRE].

[23] O. Mena and S.J. Parke, Untangling CP-violation and the mass hierarchy in long baseline experiments, Phys. Rev. D 70 (2004) 093011 [hep-ph/0408070] [InSPIRE].

[24] S.K. Agarwalla, S. Prakash, S.K. Raut and S.U. Sankar, Potential of optimized NOvA for large $\theta_{(13)}$ and combined performance with a LArTPC and T2K, JHEP 12 (2012) 075 [arXiv: 1208.3644] [INSPIRE].

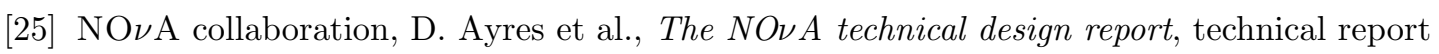
(2007).

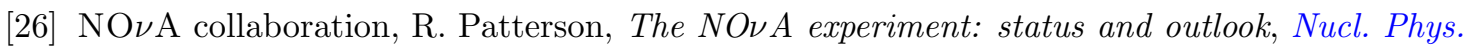
Proc. Suppl. 235-236 (2013) 151 [arXiv:1209.0716] [InSPIRE].

[27] H. Nunokawa, S.J. Parke and R. Zukanovich Funchal, Another possible way to determine the neutrino mass hierarchy, Phys. Rev. D 72 (2005) 013009 [hep-ph/0503283] [INSPIRE].

[28] P. Huber, M. Lindner and W. Winter, Simulation of long-baseline neutrino oscillation experiments with GLoBES (General Long Baseline Experiment Simulator), Comput. Phys. Commun. 167 (2005) 195 [hep-ph/0407333] [INSPIRE].

[29] P. Huber, J. Kopp, M. Lindner, M. Rolinec and W. Winter, New features in the simulation of neutrino oscillation experiments with GLoBES 3.0: General Long Baseline Experiment Simulator, Comput. Phys. Commun. 177 (2007) 432 [hep-ph/0701187] [INSPIRE].

[30] T2K collaboration, Y. Itow et al., The JHF-Kamioka neutrino project, hep-ex/0106019 [INSPIRE].

[31] Three-neutrino results after the 'TAUP 2013' conference, see webpage.

[32] F. Capozzi, G.L. Fogli, E. Lisi, A. Marrone, D. Montanino et al., Status of three-neutrino oscillation parameters, circa 2013, Phys. Rev. D 89 (2014) 093018 [arXiv:1312.2878] [INSPIRE].

[33] T2K collaboration, K. Abe et al., Precise measurement of the neutrino mixing parameter $\theta_{23}$ from muon neutrino disappearance in an off-axis beam, Phys. Rev. Lett. 112 (2014) 181801 [arXiv: 1403.1532] [INSPIRE].

[34] J.-E. Campagne, M. Maltoni, M. Mezzetto and T. Schwetz, Physics potential of the CERN-MEMPHYS neutrino oscillation project, JHEP 04 (2007) 003 [hep-ph/0603172] [INSPIRE]. 